Nature, submitted

\title{
Advances in Exoplanet Science from Kepler
}

\author{
Jack J. Lissauer, Rebekah I. Dawson,2 \& Scott Tremaine ${ }^{3}$
}

\begin{abstract}
Numerous telescopes and techniques have been used to find and study extrasolar planets, but none has been more successful than NASA's Kepler Space Telescope. Kepler has discovered the majority of known exoplanets, the smallest planets to orbit normal stars, and the worlds most likely to be similar to our home planet. Most importantly, Kepler has provided our first look at typical characteristics of planets and planetary systems for planets with sizes as small as and orbits as large as those of the Earth.
\end{abstract}

Kepler is a $0.95 \mathrm{~m}$ aperture space telescope launched by NASA in 2009 (Borucki et al. 2010; Koch et al. 2010). Kepler identifies those exoplanets whose orbits happen to appear edge-on by searching for periodic dips caused by planetary transits (partial eclipses) of the stellar discs. Above Earth's atmosphere, and in an Earth-trailing heliocentric orbit away from the glare and thermal variations of low Earth orbit, Kepler monitored the brightness of more than $10^{5}$ stars at 30-minute cadence for four years. Kepler's unique asset is an unprecedented photometric precision of $\sim 30$ parts per million $(\mathrm{ppm})$ for 12th magnitude stars with data binned in 6.5 hour intervals (Gilliland et al. 2011). This time interval is used as a benchmark because the Earth takes 13 hours to transit the Sun as viewed by a distant observer in the ecliptic plane, and observers slightly away from the ecliptic view a transit of shorter duration. Such high-precision measurements are only possible in space, where stars do not twinkle, and are required to search for Earth-like worlds because the transit of such a planet across the disc of a Sun-like star blocks only $80 \mathrm{ppm}$ of the stellar flux. For comparison, the transit of a Jupiter-size planet across a similar star blocks $1 \%$ of the flux, and this dip is straightforward to detect using ground-based telescopes.

Transits of $\sim 3600$ planet candidates, the vast majority of which represent true exoplanets as described below, have been identified in the first three years of Kepler data (Figure 11. The discovery of these worlds, most of which have orbital periods (local "years") shorter than a few Earth months, has greatly expanded the zoo of known exoplanet types. Most

\footnotetext{
${ }^{1}$ NASA Ames Research Center, Moffett Field, CA 94035, USA

${ }^{2}$ Department of Astronomy, University of California, Berkeley, CA 94720, USA

${ }^{3}$ Institute for Advanced Study, Princeton, NJ 08540, USA
} 
Kepler planets have radii, $R_{p}$, intermediate between those of Earth and Neptune $\left(1-3.8 \mathrm{R}_{\oplus}\right.$, where $\mathrm{R}_{\oplus}=6371 \mathrm{~km}$ is the Earth's radius); planets in this size range are missing from our Solar System. These planets have a wide range of densities (Batalha et al. 2011; Lissauer et al. 2011a; Doyle et al. 2011; Carter et al. 2012; Jontof-Hutter et al. 2014; Marcy et al. 2014), probably because they have atmospheres with a wide range of properties. Nonetheless, theoretical models of their interiors (e.g., Fortney et al. 2007) imply that all of the planets in this class are "gas-poor", that is, less than half — in most cases far less — of their mass consists of hydrogen and helium $(\mathrm{H} / \mathrm{He})$. In contrast, $\mathrm{H} / \mathrm{He}$ make up more than $98 \%$ of our Sun's mass as well as substantial majorities of the masses of Jupiter, Saturn, and almost all known giant exoplanets with $R_{p}>9 \mathrm{R}_{\oplus}$.

Kepler's primary mission is to conduct a statistical census of the abundance of planets as a function of planetary size, orbital period and stellar type. Kepler has found that planets are common, with the number of planets in the extended solar neighborhood of our Galaxy being comparable to or larger than the number of stars (Dressing \& Charbonneau 2013). Of particular interest is $\eta_{\oplus}$, the average number of Earth-like planets per star. "Earth-like" means having a radius similar to that of Earth and receiving about as much energy flux from its host star as Earth receives from our Sun; see below for a more precise definition. With some extrapolation downward in size and longward in orbital period, Kepler data suggest that $\eta_{\oplus} \sim 0.1$, although as discussed below, there is a broad range of estimates of this value. Previous studies (Fischer \& Valenti 2005; Sousa et al. 2011) have found giant planets to be much more common around stars that are richer in heavy elements relative to light gases; Kepler data have shown that no comparable trend exists for small planets (Buchhave et al. 2012; Wang \& Fischer 2013). Almost half of Kepler's planet candidates are in systems in which multiple transiting planets have been found. As discussed below, the large abundance of such systems implies that flat systems containing multiple planets on closely spaced orbits are quite common. This finding supports models of planet formation within a disc of material orbiting a star that date back to Kant and Laplace.

Kepler's prime mission ended in May 2013 with the failure of a second reaction wheel that made precise stable pointing away from the spacecraft's orbital plane impossible. Nevertheless, data analysis over the next few years is expected to reveal hundreds or even thousands of additional planet candidates, probably including some that extend the range of exoplanets to smaller sizes and longer periods (lower right in Figure 1), perhaps including true Earth analogs in size and period that orbit Sun-like stars. These additional planets, plus better estimates of planetary sizes and planet detectability, will allow for improved estimates of the population of planets within our Galaxy. Although the hobbled Kepler spacecraft cannot observe its original star field any longer, it has been reprogrammed to continue its search for other worlds, with a focus on planets orbiting small stars with orbital periods of less 


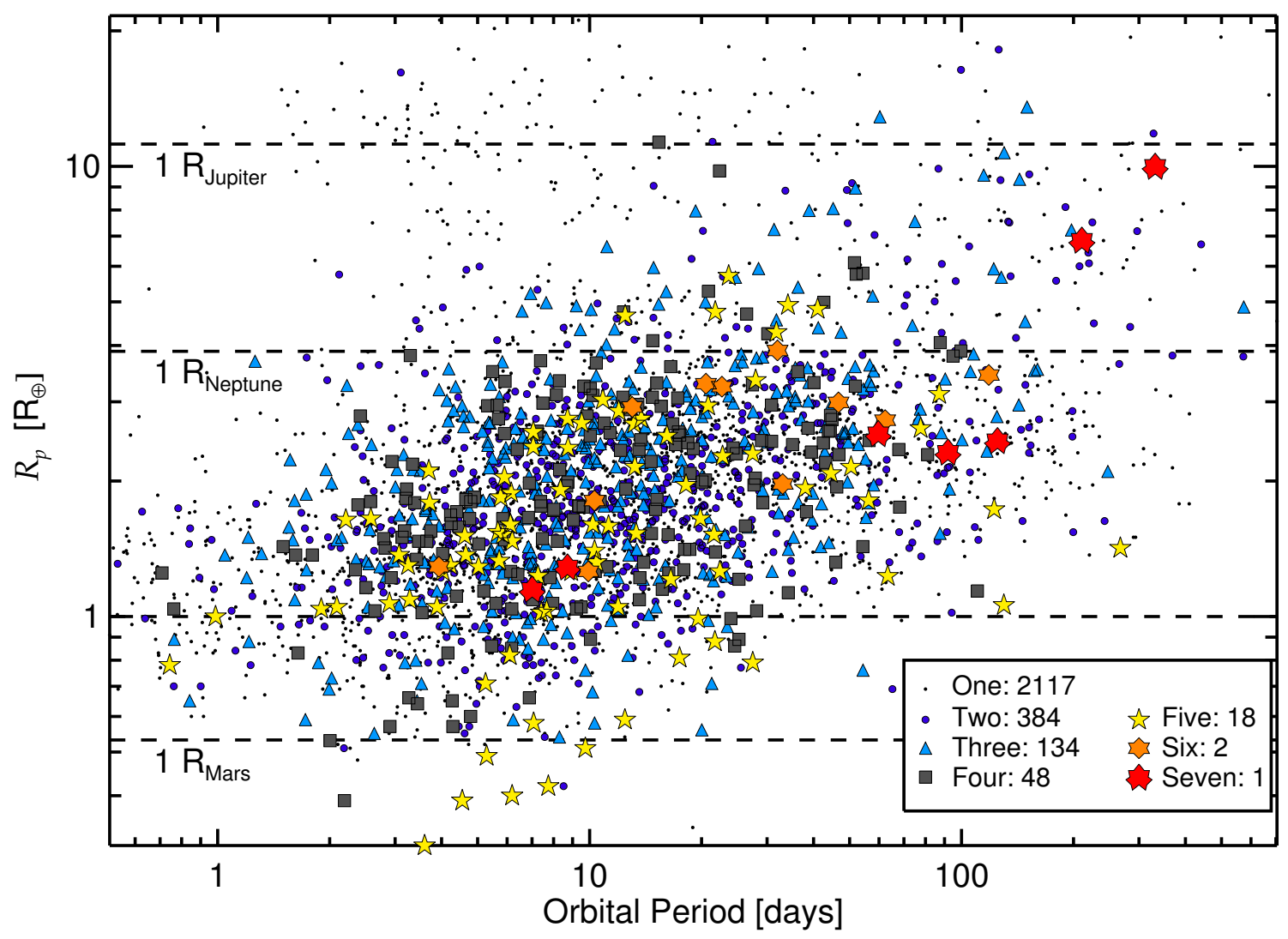

Fig. 1.- Orbital period versus planetary radius for planetary candidates found by analyzing the first three years of Kepler data. Planets are shown by coloured symbols that represent the number of candidates in the system, as indicated by the legend in the lower right. The numbers shown in the legend represent the total number of systems of a given multiplicity in the catalog; a small fraction of these planets fall outside the boundary of the period-radius ranges plotted. Planets with shorter orbital periods are over-represented because geometric factors and frequent transits make them easier to detect in Kepler data. The upward slope in the lower envelope of these points is caused by the difficulty in detecting small planets with long orbital periods, for which transits are shallow and few are observed. The apparent absence of giant planets in multi-planet systems has been quantitatively confirmed (Latham et al. 2011). Data provided by Jason Rowe.

than one month; Kepler 's new mission is dubbed "K2". Other space missions will expand and exploit Kepler's discoveries over the next decade. The European Space Agency (ESA) recently launched the Gaia astrometric spacecraft, which will determine precise distances to Kepler planet-hosting stars, enabling more accurate determination of the sizes of these stars and their associated planets. NASA's Transiting Exoplanet Survey Satellite (TESS), 
scheduled to launch in 2017, will conduct an all-sky search for transiting planets around the nearest and brightest stars using small-aperture, wide-field optics (Ricker et al. 2014). TESS planets will be easier to study with other space- and ground-based observatories than are Kepler planets, most of which orbit much fainter stars. Searches for transiting planets in space will continue in the 2020's with ESA's PLATO mission, which will have an effective aperture almost as large as does Kepler, together with a much larger field of view. Analysis of data from these advanced observatories, together with associated theoretical studies, should advance the studies of exoplanets pioneered by Kepler far beyond the mission's original goals.

\section{Transiting planets and eclipsing binary stars}

The transit depth yields the ratio of the planetary radius to the stellar radius, and the repetition rate of transits tells us the planet's orbital period. The stellar colors - or, better yet, stellar spectrum - can be used to deduce the star's radius and mass, and from these we can find the planet's radius and the semi-major axis of its orbit (from Kepler's third law). In favourable cases (generally restricted to close-in planets that are subjected to intense stellar irradiation), we can detect the occultation of the planet as it travels behind the star, and thus determine the planet's albedo (i.e., its reflectivity). A wide range of albedos is found for both small (Demory 2014) and large planets (Angerhausen et al. 2014), with most (hot) giant planets having low albedo. Planets in multiple systems perturb one another through their mutual gravity, causing their orbits to deviate from strict periodicity. These deviations

lead to transit timing variations (TTVs) that in favourable cases can be used to measure the planetary masses and additional orbital elements (Holman et al. 2010; Lissauer et al. 2011 a;

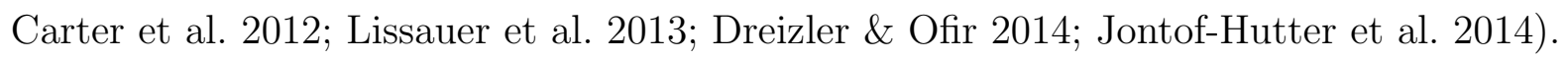

The objects in the catalogs assembled by the Kepler project Borucki et al. 2011; Batalha et al. 2013; Burke et al. 2014) are considered to be only planet candidates because eclipsing binary stars can mimic transiting planets. Normally the fractional brightness change in a binary-star eclipse is much larger than in a planetary transit, but occasionally the eclipse is grazing, or light from the Kepler target star is diluted or "blended" with the light from a background or companion eclipsing binary that is nearby on the plane of the sky. Such false positives plague ground-based searches for exoplanets, but the Kepler light curves (starlight received as a function of time) are of such high quality that they can usually be used to discriminate between grazing or blended stellar eclipses and planetary transits. Moreover, Kepler is an imaging instrument, which can measure changes in the position of the image on the sky plane during transit. This "centroiding" weeds out most eclipsing binaries that are blended with background stars and some that are blended with companion stars. Thus, 
although well under half of Kepler's planet candidates have been verified to be true planets, the false-positive rate of the catalog as a whole is probably less than $10 \%$, although it may exceed $30 \%$ for the largest planet candidates (Morton \& Johnson 2011; Santerne et al. 2012; Fressin et al. 2013). Therefore, with appropriate care, the Kepler catalog can be used for statistical studies of the exoplanet population.

Several dozen candidates not found by the Kepler data analysis pipeline have been identified by eye by members of the public through the Planet Hunters project Fischer et al. 2012), and other groups have found dozens of planets with orbital periods of $<1$ day for which the pipeline is not optimized (Sanchis-Ojeda et al. 2014).

Planet candidates that have been verified to be true planets at a high level of confidence (in most cases well above 99\%) are assigned Kepler designations (names). Verification can take the form of dynamical confirmation by detection of either TTVs in the Kepler light curve or radial-velocity variations, or it can be based on statistical arguments showing that the likelihood of the planet hypothesis is much greater than that of other possible causes of the observed light curve (Torres et al. 2011; Morton 2012; Lissauer et al. 2014).

\section{Individual planets and planetary systems}

Kepler's primary mission is a statistical characterization of the exoplanet population, but we first describe some of the highlights of individual planets and planetary systems found by Kepler.

Kepler's first major discovery was the Kepler-9 system, which contains two transiting giant planets with orbital periods of 19.24 and 38.91 days. The nearby 2:1 orbital resonance $(38.91 / 19.24=2.02)$ induces TTVs of tens of minutes in both planets. Analysis of these TTVs enabled both planets to be confirmed and provided estimates of their masses: they are similar in size to Saturn but less than half as massive (Holman et al. 2010; Dreizler \& Ofir 2014). TTVs have also been used to discover non-transiting planets, such as Kepler-19 c (Ballard et al. 2011) and Kepler-46 c (Nesvorný et al. 2012).

The first rocky planet found by Kepler was Kepler-10 b, which has $R_{p}=1.42 \pm 0.04 \mathrm{R}_{\oplus}$ and mass $M_{p}=4.6 \pm 1.2 \mathrm{M}_{\oplus}($ Batalha et al. 2011$)$, where $\mathrm{M}_{\oplus}=5.978 \times 10^{24} \mathrm{~kg}$ is the Earth's mass. This planet's density, $8.8_{-2.9}^{+2.1} \mathrm{~g} \mathrm{~cm}^{-3}$, is consistent with an Earth-like composition. Remarkably, its orbital period is only 20 hours.

Kepler-11 is a Sun-like star with six transiting planets that range in size from $\sim 1.8-$ $4.2 \mathrm{R}_{\oplus}$ (Lissauer et al. 2011a, 2013). Orbital periods of the inner five of these planets are 
between 10 and 47 days, with the ratio of orbital periods between adjacent planets ranging from 1.26 to 1.74. For comparison, the ratio of orbital periods in the Solar System ranges from 1.63 (Venus and Earth) to 6.3 (Mars and Jupiter). The outermost planet, Kepler-11 $\mathrm{g}$, has a period of 118.4 days. TTVs have been used to estimate the planets' masses. Most if not all have a substantial fraction of their volume occupied by the light gases hydrogen (in the form of molecular hydrogen) and helium, which implies that $\mathrm{H} / \mathrm{He}$ can dominate the volume of a planet that is only a few times as massive as the Earth.

Kepler-36 (Carter et al. 2012) hosts two planets whose semi-major axes differ by only $10 \%$ but whose compositions are dramatically different: rocky Kepler-36 b has a mass $M_{p}=$ $4.5 \pm 0.3 \mathrm{M}_{\oplus}$, a density of $7.46_{-0.59}^{+0.74} \mathrm{~g} \mathrm{~cm}^{-3}$ and an orbital period, $P$, of 13.84 days, whereas puffy Kepler-36 c has $M_{p}=8.7 \pm 0.5 \mathrm{M}_{\oplus}$, a density of $0.89_{-0.05}^{+0.07} \mathrm{~g} \mathrm{~cm}^{-3}$ and $P=16.24$ days. Possibly the atmosphere of Kepler-36 b was stripped by photo-evaporation or impact erosion, while Kepler-36 c was able to retain its atmosphere because of its larger core mass and slightly larger distance from the host star (Lopez \& Fortney 2013). The proximity of the orbits also presents a conundrum: while numerical integrations show that the current configuration may be long-lived, most nearby configurations are unstable on short timescales (Deck et al. 2012), so it is far from clear how these planets arrived at their current orbits.

The first transiting circumbinary planet to be discovered, Kepler-16 b, is an object of approximately Saturn's mass and radius $\left(M_{p}=106 \pm 5 \mathrm{M}_{\oplus}, R_{p}=8.27 \pm 0.03 \mathrm{R}_{\oplus}\right)$, traveling on a nearly circular orbit (eccentricity $e=0.0069$ ) with a period of 228.8 days around an eclipsing pair of stars with an orbital period of 41.08 days (Doyle et al. 2011). A bonus in such systems is that the planetary transits enable accurate measurements of the stellar masses and radii (errors $\lesssim 0.5 \%$ ): one of the stars is about two-thirds the size and mass of our Sun and the other only a fifth as large as the Sun (Doyle et al. 2011). Moreover, the primary star's rotation axis has been measured to be aligned with the binary's orbital axis to within $2.4^{\circ}$ (Winn et al. 2011). Several other circumbinary planets have been found using Kepler data, including the multi-planet Kepler-47 system (Orosz et al. 2012).

Kepler-20 e is the first planet smaller than Earth $\left(R_{p}=0.87_{-0.10}^{+0.08} R_{\oplus}\right)$ to be verified around a main-sequence star other than the Sun (Fressin et al. 2012); its 6.1-day orbit means that it is far too hot to be habitable. The low-mass (M-dwarf) star Kepler-42 hosts three validated planets smaller than Earth, the smallest of which is Mars-sized (Muirhead et al. 2012). Kepler-37 b, only slightly larger than Earth's Moon, is the first planet smaller than Mercury to be found orbiting a main-sequence star; its period is 13 days, and the stellar host is $80 \%$ as massive as the Sun (Barclay et al. 2013). KIC 12557548 b exhibits transits of varying depths, which might be due to an evaporating dusty atmosphere (Rappaport et al. 2012). Kepler-78 b (Sanchis-Ojeda et al. 2013) has the shortest orbital period of any 
confirmed exoplanet, circling its star in 8.5 hours. This roasting world is slightly larger than Earth, and its mass, measured from the radial-velocity variations it induces in its nearby host star, implies a rocky composition (Howard et al. 2013 ; Pepe et al. 2013$)$.

Circumstellar habitable zones are conventionally defined to be the distances from stars where planets with an atmosphere similar to Earth's receive the right amount of stellar radiation to maintain reservoirs of liquid water on their surfaces (Kopparapu et al. 2013). Kepler-62 $\mathrm{f}$ is the first known exoplanet whose size $\left(1.41 \pm 0.07 \mathrm{R}_{\oplus}\right)$ and orbital position suggest that it could well be a rocky world with stable liquid water at its surface (Borucki et al. 2013).

\section{Principal goals of the Kepler mission}

Discoveries like the ones mentioned above have captured a great deal of attention in the scientific community and beyond. But Kepler is, in essence, a statistical mission, designed to discover large numbers of planets in a survey with well-characterized selection criteria. The stated goals of the Kepler mission prior to launch (Borucki et al. 2007) were to explore the structure and diversity of extrasolar planetary systems and thereby to:

1. determine the frequency of Earth-size and larger planets in or near the habitable zone of a wide variety of spectral types of stars;

2. determine the distributions of size and orbital semi-major axes of these planets;

3. estimate the frequency of planets in multiple-star systems;

4. determine the distributions of semi-major axis, albedo, size, mass and density of short period giant planets;

5. identify additional members of each photometrically discovered planetary system using complementary techniques;

6. determine the properties of those stars that harbor planetary systems.

In its four-year prime mission Kepler observed a total of almost 200,000 stars, including $\sim 140,000$ dwarf or main-sequence stars that were monitored for a substantial majority of the time. In addition to its contribution to exoplanet science, Kepler has revolutionized the field of asteroseismology, which probes stellar interiors by observing the surface manifestations of oscillations that propagate within stars (Chaplin \& Miglio 2013; Christensen-Dalsgaard 
2013), and has dramatically advanced our understanding of eclipsing binary stars (Prsa et al. 2011) as well as other areas of stellar physics. This article only considers stellar properties indirectly through their contributions to assessing planetary characteristics.

\section{How common are planets?}

The Kepler catalog of planets is uniquely valuable for studying the structure and properties of planetary systems: it is large enough that we can map out the distribution of planets in multiple parameters (orbital period, radius, multiplicity, properties of the host star, etc.); it has, at least in principle, well-defined selection criteria (in contrast to radial-velocity catalogs, which come from many different surveys and which usually do not include null results); and most of the parameter space that it explores - typical radii of $1-3 R_{\oplus}$ and orbital periods up to $\sim 1$ year (Figure 1 ) — is not easily accessible by other techniques.

One of the most fundamental statistics describing planetary systems is the probability distribution $f\left(R_{p}, P\right) d \ln R_{p} d \ln P$ that a member of a specified class of stars possesses a planet in the infinitesimal area element $d \ln R_{p} d \ln P$ (Tabachnik \& Tremaine 2002; Youdin 2011; Howard et al. 2012; Dong \& Zhu 2013). The integral of this distribution over a range in planetary radius and orbital period is the average number of planets per star (not to be confused with the fraction of stars with planets, which is smaller). Kepler determines this distribution for solar-type stars with reasonable accuracy for $R_{p} \gtrsim 1 \mathrm{R}_{\oplus}$ in the range $P \lesssim 50$ days, and for $R_{p} \gtrsim 2 \mathrm{R}_{\oplus}$ in the range $P \lesssim 150$ days.

Occurrence rate calculations must carefully account for the completeness, reliability, and threshold criteria of the Kepler catalogue (Jenkins et al. 2010; Tenenbaum et al. 2012), as well as random and systematic errors in the host-star properties. For candidates with small transit depths or just a few transits, robust estimates of occurrence rates require calibration by injecting and recovering planetary signals in Kepler data (Christiansen et al. 2013, Petigura \& Marcy 2012; Petigura et al. 2013a.b). The false-positive frequency distribution must be modeled simultaneously (Fressin et al. 2013). Revisions of host-star properties can dramatically alter the radius distribution of planets (Dressing \& Charbonneau 2013). No occurrence rate calculations to date contain all of these ingredients. Here we summarize key results from early analyses of the Kepler dataset (see Batalha 2014 for a review).

The studies cited above find that the number of planets per unit log period is nearly flat for $R_{p} \lesssim 4 \mathrm{R}_{\oplus}$ and $P>10$ days, but rises by a factor $2-5$ between $P=10$ days and $P=100$ days for larger planetary radii. The number of planets drops sharply for orbital periods below 10 days for $R_{p} \lesssim 4 \mathrm{R}_{\oplus}$ and below $2-3$ days for giant planets. The occurrence rate of giant 
planets on small orbits is a factor of three lower than in radial-velocity surveys (Howard et al. 2012; Wright et al. 2012; Fressin et al. 2013), perhaps because a significant fraction of giant planets are injected into small orbits through planet-planet gravitational interactions, and the relatively metal-poor Kepler stars host fewer and/or less massive planets, which are less likely to interact strongly (Dawson \& Murray-Clay 2013). At all periods the number per $\log$ radius grows as the radius shrinks, at least down to radii of $2 \mathrm{R}_{\oplus}$. Below $2 \mathrm{R}_{\oplus}$, the distribution per unit log radius plateaus at orbital periods out to 50 days and probably out to 100 days (Dong \& Zhu 2013; Petigura et al. 2013b). The average number of planets per star with $P<50$ days is $\approx 0.2$ for $1 \mathrm{R}_{\oplus}<R_{p}<2 \mathrm{R}_{\oplus}$ and $\approx 0.4$ for all radii $R_{p}>1 \mathrm{R}_{\oplus}$ (Dong \& Zhu 2013; Petigura et al. 2013a). For stars cooler and less massive than the Sun, the average number of planets per star is even higher (Dressing \& Charbonneau 2013): $0.49_{-0.05}^{+0.07}$ for $1 \mathrm{R}_{\oplus}<R_{p}<2 \mathrm{R}_{\oplus}$ and $0.69_{-0.06}^{+0.08}$ for all radii $R_{p}>1 \mathrm{R}_{\oplus}$ with $P<50 \mathrm{~d}$. The higher frequency is remarkable since the fixed period cutoff at $50 \mathrm{~d}$ corresponds to a smaller semi-major axis in the less massive stars.

A widely used milestone is $\eta_{\oplus}$, the number of Earth-like planets of Sun-like stars. One difficulty in discussing $\eta_{\oplus}$ is that different authors use different definitions for "Earth-like". For solar-type stars the most natural definition is $\eta_{\oplus}=f\left(1 \mathrm{R}_{\oplus}, 1 \mathrm{yr}\right)$; for other stars we can replace $P=1$ yr with the period corresponding to the same incident stellar flux. Roughly speaking, this is the number of planets per star in a range of a factor of $e$ in radius and period centered on the Earth's radius and period. Unfortunately, determining $\eta_{\oplus}$ according to this definition requires an extrapolation downwards in size and longward in orbital period from the region where Kepler has a statistically reliable planet sample, which introduces considerable uncertainty. Applying this extrapolation to power-law fits $f\left(R_{p}, P\right) \propto P^{\beta}$ (Dong \& Zhu 2013) of the distribution of planets in the 16-month Kepler catalog (Batalha et al. 2013) yields $\eta_{\oplus}=0.09$. An independent analysis of Kepler light curves (Petigura et al. 2013b) gives a consistent result, $\eta_{\oplus}=0.12 \pm 0.04$, after renormalizing by a factor of 2.1 to convert their definition to ours. However, a follow-up study using the same catalogue but a more general form for the period and radius distribution found a much smaller value $\eta_{\oplus}=0.02_{-0.01}^{+0.02}$ (Foreman-Mackey et al. 2014). There are also other uncertainties: for example, none of the results for $\eta_{\oplus}$ discussed here model false positives (for planets of this size, the biggest contributor is larger planets orbiting fainter stars that appear close to the Kepler target on the plane of the sky; Fressin et al. 2013). Moreover, the extrapolations involve a mix of planets with and without substantial volatile envelopes (see below), as well as a likely mix of formation histories, and therefore the extrapolation may not capture the true occurrence rate.

A related number for cool, low-mass stars is $0.16_{-0.10}^{+0.0 .14}$ planets per star with $0.5 \mathrm{R}_{\oplus}<$ $R_{p}<1.4 \mathrm{R}_{\oplus}$ receiving $0.46-1$ times the solar flux at Earth (Dressing \& Charbonneau 2013), 
which corresponds to a rate of $0.26_{-0.16}^{+0.23}$ in an interval equal to that we use to define $\eta_{\oplus}$.

\section{The diverse physical properties of Kepler planets}

Kepler has discovered more than 3000 planet candidates with radii $R_{p}<4 \mathrm{R}_{\oplus}$. Planetary interior models show warm planets of this size to be "gas-poor," defined here as composed of less than $50 \% \mathrm{H} / \mathrm{He}$ by mass. Transit surveys are well-suited to studying the physical properties of such planets because their radii are very sensitive to small amounts of gas in the atmosphere - for example, just $1 \% \mathrm{H} / \mathrm{He}$ added to a $1 \mathrm{M}_{\oplus}, 1 \mathrm{R}_{\oplus}$ solid core can inflate the planet to $2 \mathrm{R}_{\oplus}$ (Lopez \& Fortney 2014) - and moderately sensitive to the bulk composition of the core (e.g., water vs. rock). Furthermore, the gas-poor planets found by Kepler are valuable because: (i) they sample a wide range of incident fluxes and therefore were presumably subject to a wide range of photo-evaporation rates; (ii) many are found at short orbital periods, allowing mass measurements or meaningful upper limits through radial-velocity follow-up studies (Marcy et al. 2014), and (iii) some are found in compact systems with multiple transiting, low-density planets, whose short orbital periods and large radii allow sensitive mass measurements through TTVs.

Figure 2 shows the masses, radii and incident flux received by well-characterized planets less than 20 times as massive as Earth. The wide range in size of gas-poor planets of a given mass indicates a diversity of composition. Note that most of the sub-Saturn exoplanets whose masses and radii are both known are Kepler discoveries.

Various processes can affect the composition of gas-poor planets, including coagulation from volatile-rich or volatile-poor planetesimals, accretion of gas from the proto-planetary nebula if the planet forms before its dispersal, outgassing of volatiles from the planet's interior, atmospheric escape (e.g., via photo-evaporation), and erosion or enrichment of the atmosphere and mantle via collisions with planetesimals. Distinguishing which of these processes are at play and their relative contributions is both a challenge and a motivation for interpreting the measurements from Kepler.

Several of the planets discussed earlier and highlighted in Figure 2 have served as case studies to illuminate the properties of gas-poor planets. In particular, they sample a continuum of photo-evaporation rates, which are a function of both incident stellar flux (as a

proxy for the X-ray/UV radiation responsible for atmospheric erosion) and core mass. The middle four planets of Kepler-11, each of which contains $\sim 4-15 \% \mathrm{H} / \mathrm{He}$ by mass, might represent the pristine, uneroded initial compositions of gas-poor planets, whereas the innermost planet in the system, Kepler- $11 \mathrm{~b}$, is only $0.5 \% \mathrm{H} / \mathrm{He}$ by mass (or maybe devoid of 


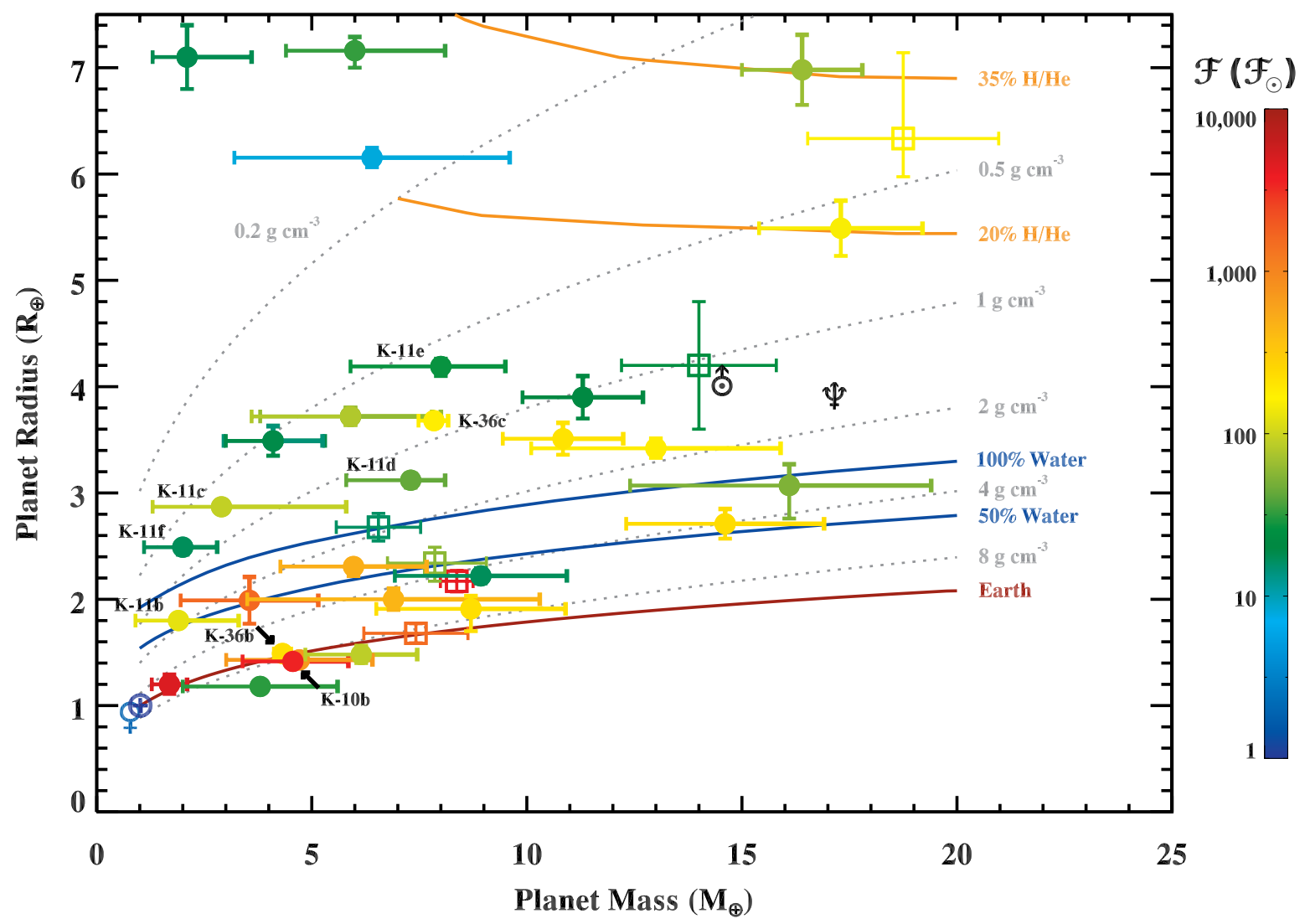

Fig. 2.- Mass-radius diagram for transiting exoplanets with measured masses less than $20 \mathrm{M}_{\oplus}$, along with model curves for different compositions. Planets are color-coded by the incident bolometric flux they receive. Kepler planets are shown by filled circles, with numbers and letters indicating planets discussed in the text; the rocky planets in the crowded region near the lower left include Kepler-10 b (red point) and Kepler-36 b (yellow). Other known exoplanets in this mass range are shown by open squares. The Solar System planets Venus, Earth, Uranus and Neptune are shown by their symbols. The lower curve is for an Earth-like composition with $2 / 3$ rock and $1 / 3$ iron by mass. All other curves use thermal evolution calculations (Lopez et al. 2012), assuming a volatile atmosphere of $\mathrm{H} / \mathrm{He}$ or water atop a core of rock and iron with composition the same as that of the bulk Earth. The two blue curves are for $50 \%$ and $100 \%$ water by mass and the two orange curves are for $\mathrm{H} / \mathrm{He}$ atmospheres atop Earth-composition cores. These theoretical curves assume a radiation flux 100 times as large as that received by Earth and an age of 5 Gyr. Figure courtesy of Eric Lopez.

light gases entirely if it is water-rich), perhaps because it has undergone significant mass loss from its primordial atmosphere (Lissauer et al. 2013). Kepler-10 b, with an incident flux 
about 30 times that of Kepler-11 b, may in turn have lost all of its atmosphere to photoevaporation. This speculation is based on Kepler-10 b's density, which can be matched by theoretical models that do not require a volatile component (Batalha et al. 2011). In addition to lifetime-integrated X-ray and UV flux, core mass is an important factor in determining the photo-evaporation rate. A larger core mass for Kepler-36 c may have enabled it to maintain its atmosphere against photo-evaporation, which may have stripped its nearby neighbour Kepler-36 b (Lopez \& Fortney 2013).

There is now a large collection of gas-poor Kepler planets with masses that are individually less precisely measured than the case studies above but nonetheless statistically powerful when analyzed as an ensemble. Dozens of masses have been measured via radial-velocity follow-up (Marcy et al. 2014). Using an approach that accounts for degeneracies between mass and eccentricity (Lithwick et al. 2012), over 100 were estimated from TTVs (Hadden \& Lithwick 2014). Furthermore, theoretical models imply that radii of warm planets in the size range $2-4 \mathrm{R}_{\oplus}$ depend far more on $\mathrm{H} /$ He percentage than on total planet mass (Lopez \& Fortney 2014). Thus, the larger sample of thousands of Kepler candidates with $R_{p}<4 \mathrm{R}_{\oplus}$, even if lacking measured masses, informs us about planetary occurrence rates as a function of composition and their correlations with other properties such as the orbital period and the mass and chemical composition of the host star, which in turn constrain models for the formation and evolution of gas-poor planets. Several radius ranges may indicate different regimes for planet formation and evolution, as illustrated in Figure 3 :

- $R_{p} \lesssim 1.6 \mathrm{R}_{\oplus}$ : Most of the small number of transiting planets in this radius range that have measured masses are dense enough to be rocky; in contrast, larger planets appear to require a volatile component (Rogers 2014). This radius range includes all verified Kepler planets with period $P<1$ day (Sanchis-Ojeda et al. 2014); these planets may once have possessed atmospheres that have now been stripped by impacts, photo-evaporation, stellar winds, and/or tidal forces.

- $1.6 \mathrm{R}_{\oplus} \lesssim R_{p} \lesssim 3 \mathrm{R}_{\oplus}$ : In this regime, the mass-radius relation is consistent with $M_{p} \propto$ $R_{p}$, indicating that the typical planetary density decreases with increasing size $\mathrm{Wu} \&$ Lithwick 2013). This mass-radius relation requires a substantial mass fraction of water or a small mass fraction $(0.1-5 \%)$ in a $\mathrm{H} / \mathrm{He}$ atmosphere $\mathrm{Wu} \&$ Lithwick 2013; Weiss \& Marcy 2014). The scatter in the mass-radius relation exceeds the measurement errors (Weiss \& Marcy 2014) indicating some diversity in composition and/or atmospheric properties, possibly including rare rocky planets without voluminous atmospheres. The presence of a $\mathrm{H} / \mathrm{He}$ atmosphere substantially increases the temperature at the rocky surface; thus planets such as Kepler-22 b, which has a radius of $2.4 \mathrm{R}_{\oplus}$ (Borucki et al. 2012), are unlikely to be habitable. 


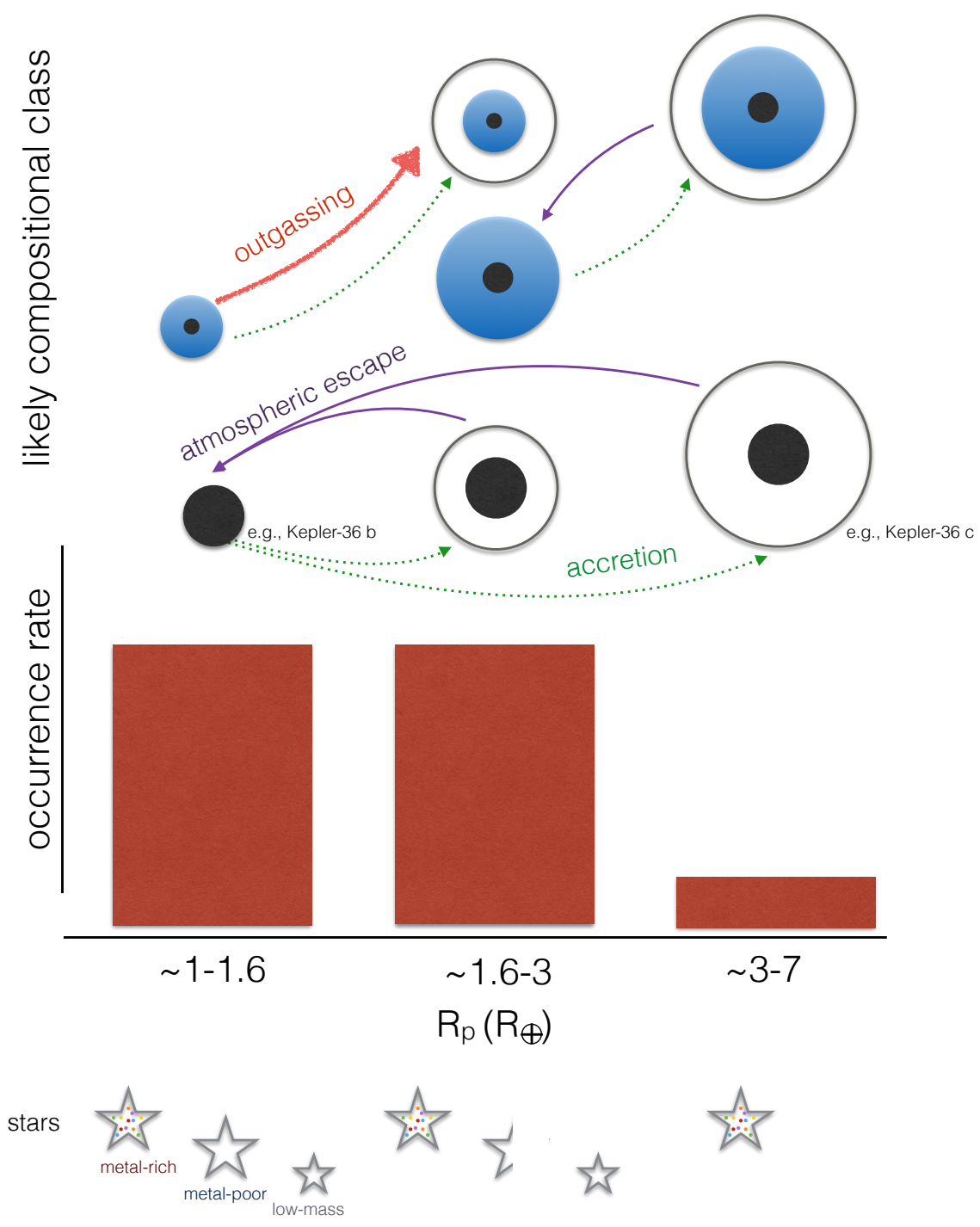

Fig. 3.- Schematic illustration of plausible compositions of the small and mid-sized planets observed by Kepler, including rock (dark grey), water (blue), and light gases (white). The red bars indicate their approximate relative occurrence rates (Dong \& Zhu| 2013; Fressin et al. 2013; Petigura et al. 2013b), and the arrows indicate physical processes that set or alter planet compositions. The smallest planets, on the left, can be rocky or possibly mixtures of rock and $\mathrm{H}_{2} \mathrm{O}$. Somewhat larger planets have volumetrically significant amounts of constituents less dense than rock. Planets whose sizes are comparable to or larger than that of Neptune, $R_{p}=3.8 \mathrm{R}_{\oplus}$, have envelopes composed of the lightest gases, $\mathrm{H}_{2}$ and He. 
- $3 \mathrm{R}_{\oplus} \lesssim R_{p} \lesssim 7 \mathrm{R}_{\oplus}$ : Planets in this size range are less dense than water, implying voluminous $\mathrm{H} / \mathrm{He}$ atmospheres (Wu \& Lithwick 2013). The occurrence rate plummets between 2 and $3 \mathrm{R}_{\oplus}$ (Petigura et al. 2013b; Morton \& Swift 2014), so this class is much rarer than the first two. Few planets in this class have been found around low-mass stars (Wu \& Lithwick 2013).

- $R_{p} \gtrsim 4 \mathrm{R}_{\oplus}$ : Large planets are more common around stars with larger abundances of elements heavier than helium (Buchhave et al. 2012; Wang \& Fischer 2013).

The class of rocky planets with $R_{p} \lesssim 1.6 \mathrm{R}_{\oplus}$ may lack gaseous atmospheres either because they were insufficiently massive to accrete significant amounts of light gases in the regions of the protoplanetary disk where they formed and never outgassed an atmosphere, or because their primordial atmospheres were removed by giant impacts or photo-evaporation. A possible explanation for the plunge in occurrence rate between 2 and $3 \mathrm{R}_{\oplus}$ is that larger worlds need $\mathrm{H} / \mathrm{He}$ envelopes, which are uncommon, but most low-density smaller planets contain substantial water components or (very low mass) outgassed $\mathrm{H}_{2}$ envelopes. The paucity of planets larger than $3 \mathrm{R}_{\oplus}$ around low-mass stars and the higher heavy-element abundance in the host stars of systems containing planets larger than $4 \mathrm{R}_{\oplus}$ may both reflect the difficulty of accreting $\mathrm{H} / \mathrm{He}$ envelopes in a protoplanetary disc with a low surface density of solids.

\section{Properties of planetary systems}

The Kepler catalog is particularly important because it contains many multiple-planet systems, roughly eight times as many as all radial-velocity surveys combined (Burke et al. 2014). Multiple systems are expected to have a very low false-positive rate $(\lesssim 1 \%)$, because background binary-star eclipses may mimic the light curve from a single transiting planet but are unlikely to imitate two or more (Lissauer et al. 2012, 2014). Multiple systems are also valuable because gravitational interactions among the planets lead to TTVs that in some cases allow us to determine the masses and orbital properties of one or more of the planets (Holman et al. 2010; Lissauer et al. 2011a; Carter et al. 2012; Lissauer et al. 2013; Dreizler \& Ofir 2014; Jontof-Hutter et al. 2014).

Multiple systems also allow us to constrain the average mutual inclinations of the planetary orbits, either by comparing relative transit durations and orbital periods (Fabrycky et al. 2014) or by comparing the frequencies of systems with different multiplicities in the Kepler survey to those in radial-velocity surveys (since the chance that multiple planets in a single system will transit is much higher if their mutual inclination is low; Tremaine \& 
Dong 2012). Such studies show that the typical mutual inclinations in Kepler planets are only a few degrees, similar to the Solar System. Another probe is the mutual inclination between a transiting planet and its non-transiting perturber, which is not biased towards low mutual inclinations by the selection effects that are present in multi-transiting systems; the first such systems with good constraints have been found to be flat (Nesvorný et al. 2012, Dawson et al. 2014).

The finding that typical Kepler multi-planet systems are flat is perhaps the first direct evidence that most planetary systems formed from a rotating thin disc of gas and dust, as suggested by Laplace over two centuries ago. But even this uncontroversial result leads to tension with other observations. In most formation models, planets have mean inclinations that are at least half as large as the mean eccentricities (e.g., Ida 1990), and this result also holds for the planets in the Solar System, the asteroids, and the Kuiper belt. Thus we expect the mean eccentricity of the Kepler planets to be no more than about 0.1. Unfortunately, attempts to measure the eccentricity distribution of Kepler planets have been complicated by (or have sometimes brought to light) systematic uncertainties in the stellar properties (Moorhead et al. 2011; Plavchan et al. 2014; Kipping 2014), though individual constraints have been possible for a subset of well-characterized stars with high signal-to-noise transits (Kipping et al. 2012b; Dawson \& Johnson 2012). In contrast, the eccentricities of radialvelocity planets are straightforward to measure, and the mean eccentricity for those having orbital periods larger than 10 days is 0.26 , far larger than we would expect from the arguments above. Are the eccentricities and inclinations of the radial-velocity planets larger than those of the Kepler planets? Or perhaps just of those planets in Kepler's multiple planet systems? And if so, why? Might the eccentricities be over-estimated (Zakamska et al. 2011)? Or could exoplanets have much larger eccentricities than inclinations (Rafikov \& Slepian 2010)?

An equally serious tension is revealed by ground-based measurements of the stellar obliquity, the angle between the equator of the host star and the orbital plane of a transiting planet. About 80 obliquities - or at least their projections on the sky plane - have been determined, mostly through measurements of the Rossiter-McLaughlin effect (Albrecht et al. 2012). Almost half of the measured projected obliquities exceed $20^{\circ}$ and $15 \%$ exceed $90^{\circ}$; of course the width of the distribution of true (as opposed to projected) obliquities must be even larger. This result is quite different from the expectation for a Laplace-type model, in which the host star and planets form from a single rotating gas disc and thus should have a common spin and orbital axis. One possibility is that the close-in giant planets arrived on their present orbits via high-eccentricity migration, which excites large obliquities (Fabrycky $\&$ Tremaine 2007). Another possibility is that the stellar spin is misaligned with the axis of the planetary disc, perhaps because of a collision with a giant planet on a highly eccentric 
orbit or twisting of the planetary disc by external torques (Tremaine 1991; Heller 1993; Batygin 2012). To complicate the situation further, most of the handful of Kepler planets for which measurements are available, including multi-planet systems, have obliquities near zero (Sanchis-Ojeda et al. 2012; Chaplin \& Miglio 2013; Hirano et al. 2014).

The properties of multi-planet systems are constrained by the requirement that they be dynamically stable over timescales comparable to the lifetime of the star. Rigorous stability criteria are not usually available except for two-planet systems (Gladman 1993), but a useful approximate criterion is that systems composed of planets on nearly circular, nearly coplanar orbits are stable over $N \gg 1$ orbits if the separation in semi-major axis between adjacent planets, $a_{i+1}-a_{i}$, exceeds some constant $K_{N}$ times the mutual Hill radius (the separation at which the mutual planetary gravity equals the difference in the pull of the star on the two planets),

$$
R_{H, i, i+1} \equiv\left(\frac{M_{i}+M_{i+1}}{3 M_{\star}}\right)^{1 / 3} \frac{a_{i}+a_{i+1}}{2},
$$

where $M_{i, i+1}$ are the planet masses and $M_{\star}$ is the mass of the host star. For $N=10^{10}$, $K_{N} \approx 9-12$ (Smith \& Lissauer 2009). As one would expect, most of the Kepler multiplanet systems are safely stable by this criterion (see Figure 4), and numerical integrations assuming initially circular, coplanar orbits confirm that virtually all of them are stable for at least $10^{10}$ orbits (Lissauer et al. 2011b; Fabrycky et al. 2014). These results depend on the assumed mass-radius relation, but are relatively insensitive to it since the planet mass enters the definition of the Hill radius to the $1 / 3$ power.

A deeper question is whether these systems are dynamically "full" or "packed", which we define to mean that no additional planets, even with very small masses, could be inserted between the existing ones in a stable orbital configuration. The situation in our own Solar System is ambiguous: the region from Jupiter to Neptune is packed, or nearly so (Holman 1997), but inside Mercury and between Earth and Mars there are significant bands in semimajor axis where additional low-mass planets would be stable for at least $10^{8}$ years Evans \& Tabachnik 2002).

Dynamically packed systems are a natural consequence of models in which planets grow hierarchically, since planet growth should stop once all of the orbits are stable. However, the correspondence is not exact, since stable zones may not be occupied if the planets they contained collided in the final stages of hierarchical growth; moreover, the system may contain additional planets that are not transiting or fall below the Kepler detection threshold. The evidence from Figure 4 suggests that the known Kepler planets are typically separated by about twice the distance required for stability, but given the possible presence of undiscovered planets and the destabilizing effects of non-zero eccentricity, many of these 


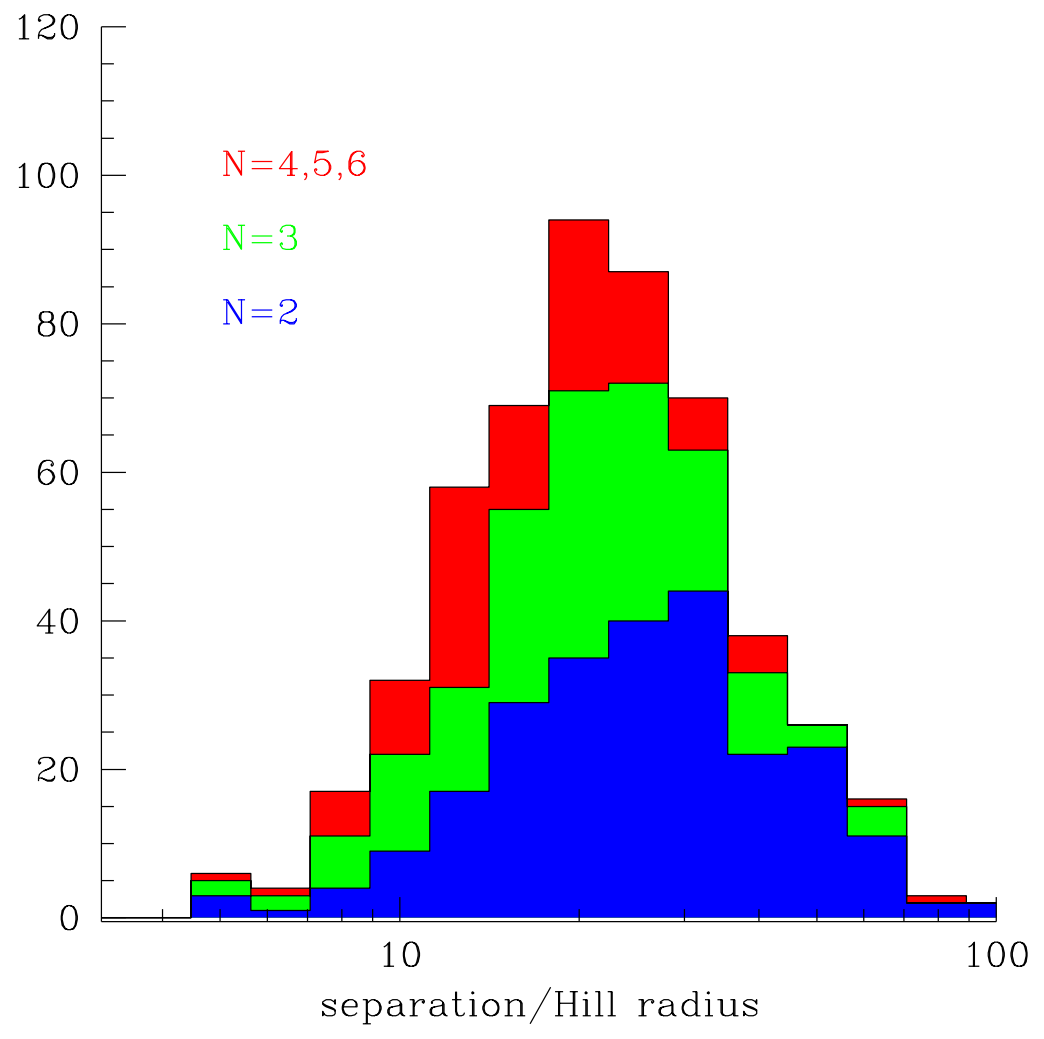

Fig. 4.- Separations of nearest neighbours in the Kepler multi-planet systems, measured in Hill radii (eq. 1), from the catalogue of Fabrycky et al. (2014). Masses are derived using the mass-radius relation $M_{p}=\mathrm{M}_{\oplus}\left(R / \mathrm{R}_{\oplus}\right)^{\alpha}$ with $\alpha=3$ for $R_{p}<\mathrm{R}_{\oplus}$ and $\alpha=2.06$ for $R_{p}>\mathrm{R}_{\oplus}$. Planets on circular, coplanar orbits separated by more than $9-12$ Hill radii are expected to be stable for the lifetime of typical stars; the few planet candidates seen with smaller separations may have large errors in their estimated radii, may not obey the assumed mass-radius relation, or may not be planets orbiting the same star.

systems may be dynamically packed (Fang \& Margot 2013).

Planets are also found in binary star systems, either orbiting one of the two stars with an orbital period much shorter than the binary period ("S-type") or orbiting both with a period much longer than the binary period ("P-type" or "circumbinary"). Most of our understanding of S-type planets comes from radial-velocity studies, whereas circumbinary 
planets around normal stars were first discovered by Kepler. In most respects the properties of planetary systems in single and binary-star systems are similar (Eggenberger 2010; Raghavan et al. 2010), although S-type planets appear to be less common in binary systems than similar planets around single stars (Wang et al. 2014).

Binary systems offer unique insights into the formation of both planets and stars. (i) Torques from the companion star in an S-type binary can excite slow, large-amplitude LidovKozai oscillations in the eccentricity and inclination of the planetary orbit. One striking hint that Lidov-Kozai oscillations are sometimes at work is that the four planets with the largest eccentricities $(e>0.85)$ are all members of wide S-type binary systems (Tamuz et al. 2008). (ii) A close companion star truncates the protoplanetary disc and the planetary system at a radius of about $0.25-0.3$ times the companion's separation (for equal-mass stars on a circular orbit, Holman \& Wiegert 1999). No S-type planets have been discovered, either in radialvelocity or transit surveys, in binary systems with separation less than about 10 times the Earth-Sun distance. This could mean that the site of planet formation in the protoplanetary disc is beyond $0.3 \times 10=3$ times the Earth-Sun distance, consistent with theories in which planets found at smaller radii have migrated inward; alternatively, the outermost regions at which circumstellar orbits are stable may nonetheless be too perturbed for planets to form. (iii) If binary stars form through dynamical interactions between single stars in a dense gas-free cluster, it would be difficult for them to acquire circumbinary planets. On the other hand, if binaries form through fragmentation and collapse in a gas-rich environment, they are likely to acquire a circumbinary disc in which planets could form. (iv) Binary stars with orbital periods of a few days are likely to be formed from binaries with much longer periods through high-eccentricity migration induced by a tertiary companion (Fabrycky \& Tremaine 2007). This process would probably remove or destroy any planets initially orbiting one of the two stars and is unlikely to produce circumbinary planets detectable by Kepler ; therefore we should not expect to find planets, S-type or P-type, in binary systems with periods of a few days or less, and this expectation is so far confirmed by the observations the shortest-period planet-hosting binary star is Kepler-47, with a period of 7.45 d (Orosz et al. 2012).

Just as important as the discoveries made by Kepler are its non-discoveries. So far Kepler has found no co-orbital planets, planets sharing the same average semi-major axis like the Trojan asteroids found accompanying Jupiter and the Saturnian satellites Janus and Epimetheus. It has found neither exomoons nor "binary" planets orbiting one another (Kipping et al. 2012a, 2013). 


\section{Planet formation}

The mass fraction of stellar material other than the dominant elements of hydrogen and helium (the metallicity, in astronomical parlance) ranges from a few percent down to $\sim 0.01 \%$ among stars in the solar neighbourhood. The initial protostellar disc presumably has the same composition as its host star, and these heavier elements are the raw material from which most of the mass in a typical Kepler planet must be drawn. Thus it is natural to expect that planet formation should be easier around stars having high metallicity. This expectation is confirmed for the giant planets detected in radial-velocity surveys: the fraction of highmetallicity stars hosting giant planets is much larger than the fraction of low-metallicity stars (Santos et al. 2001, 2004; Fischer \& Valenti 2005; Sousa et al. 2011). Similarly, a star in the Kepler catalog with super-solar metallicity is $\sim 2.5$ times more likely to host a large planet $\left(R_{p}>5 \mathrm{R}_{\oplus}\right)$ than a star with sub-solar metallicity (Wang \& Fischer 2013). Remarkably, there is no such correlation for small planets $\left(R_{p}<2 \mathrm{R}_{\oplus}\right)$ : the probabilities that a Kepler star with super-solar or sub-solar metallicity hosts a small planet are approximately equal (Wang \& Fischer 2013), with a significant number of small Kepler planets found around stars with metallicity as small as one-quarter that of the Sun (Buchhave et al. 2012). Perhaps this is a hint that the formation process for small planets has more than enough metals to draw on even in moderately low metallicity discs. Before any firm conclusions are drawn, we need reliable metallicities for a larger sample of Kepler stars and planet-frequency measurements for stars with a wider range of metallicities.

One of the most basic questions about the Kepler planets is whether they formed in situ Chiang \& Laughlin 2013, Hansen \& Murray 2013) or migrated to their current orbits from larger radii (Rogers et al. 2011; Swift et al. 2013). The orbital periods of most of Kepler's planets are $\lesssim 50$ days, resulting in nominal formation timescales that are much shorter than the lifetime of the gas disc, unlike those of the Solar System's terrestrial planets. Thus the planets and their gaseous envelopes could have formed in situ. The main argument for migration is that it is a robust process (Goldreich \& Tremaine 1980) that inevitably occurs in both analytic models and numerical simulations of planets orbiting in gaseous discs (Baruteau et al. 2013). On the other hand, models of migration have not successfully predicted any populations of planets before they were observed.

Additional insight into the migration process comes from planets in mean-motion resonances. In the strongest of these, the orbital periods of the two resonant planets are in the ratio $(n+1): n$, where $n$ is an integer. Planets can be captured permanently into resonance if they cross the resonance during migration and the migration is convergent, i.e., in a direction

such that the period ratio evolves towards unity, rather than away. Capture into resonance during convergent migration is certain if the migration is slow enough and the planetary 
eccentricities are small enough (Peale 1976). It is therefore striking that the multi-planet systems discovered by Kepler contain very few resonant planet pairs: the excess fraction of planet pairs in the Kepler sample having period ratios within $5-10 \%$ of 3:2 or 2:1 is less than 5\%. Possible explanations for the small fraction of resonant planets include the following: (i) migration was too fast for capture to occur; however, this requires migration times of $\lesssim 10^{3}$ years, far shorter than is plausible with disc migration (Rein 2012); (ii) stochastic torques on the migrating planet, which might arise in a turbulent protoplanetary disc (Rein 2012), allowed the planets to escape the resonances and continue migrating; (iii) eccentricity damping from the protoplanetary disc led to escape from the resonance (Goldreich \& Schlichting 2014); this mechanism requires that the Kepler planets have very small eccentricities, since eccentricities are difficult to excite after migration is complete (Petrovich et al. 2014); (iv) perhaps the planets formed in situ rather than migrating. A possible clue to the answer, still poorly understood, is that the distribution of period ratios in the Kepler multi-planet systems is asymmetric around the strong 2:1 and 3:2 resonances, with a peak just outside the resonance and/or a valley inside (Fabrycky et al. 2014; Lithwick \& Wu 2012; Petrovich et al. 2013).

\section{Unsolved problems}

Kepler represents a watershed in our understanding of extrasolar planets and a great stride forward in understanding the properties of planetary systems and the problems in developing theories of their formation. Yet there are many aspects of planetary systems that Kepler has not illuminated at all. Kepler has opened up a new region in the orbital period vs. radius plane, containing planets as small as Earth's Moon at short periods, and larger planets with orbital periods as large as $1-2$ years, but all of the planets in the Solar System lie outside this region (though only just outside in the case of Venus and Earth). The atmospheres of giant planets must be investigated through transit observations by ground-based telescopes or the Hubble and Spitzer space telescopes, as Kepler has no spectral resolution. The eccentricities of planetary orbits provide important insights into their formation, but only ground-based radial-velocity surveys can routinely measure eccentricities. These are expensive because the Kepler host stars are so faint, and often impossible with current technology because of the small masses of typical Kepler planets. Kepler cannot detect multiple transits of planets with periods longer than a few years, so the region beyond a few times the Earth-Sun distance, where most giant planets are likely to be born, is no better understood now than before. Planets are found occasionally at much larger radii and many have probably been ejected into interstellar space, and these regions can only be investigated by high-resolution imaging and gravitational microlensing. 
Kepler has hugely advanced our understanding of the phenomenology of exoplanets, but so far has led us no closer to a secure theory of planet formation. Did the Kepler planets form in situ or did they migrate from larger radii? Why are small planets common around host stars with such a wide range of metallicities? How did the Kepler planets acquire their voluminous atmospheres, and why are the atmospheres so diverse in mass fraction and composition? How are the Kepler planets related to the terrestrial planets in the Solar System? Why does the typical inclination of the (small) Kepler planets appear to be much less than the typical eccentricity of the (large) radial-velocity planets? How are the large angles between some planetary orbital planes and the host-star equators generated? What is the relationship between the dynamics and formation of small, rocky planets and gas-giant planets (Schlaufman 2014)?

\section{After Kepler}

Although data acquisition by the Kepler spacecraft on its original target field has ended, ongoing data analysis and observational follow-up will refine the results obtained already and address some of the outstanding questions reviewed here.

Better models of the stellar and instrumental noise in the transit light curves, including a rigorous treatment of temporally correlated noise, may enable the discovery of smaller, longer

period planets (Smith et al. 2012; Stumpe et al. 2012) as well as better characterization of existing ones. More accurate transit times are crucial given that the majority of Kepler planet masses are derived from TTVs, and more secure detections of and upper limits on transit duration variations (TDVs) will provide important constraints on mutual inclinations.

We can hope to address some of the unanswered questions about occurrence rates and system architectures by more sophisticated statistical analyses. The most complete view of the architectures of Kepler planetary systems will require tying together constraints from occurrence rates, transit durations, TTVs, and TDVs. Even the absence of TTVs can provide important constraints on planetary system architectures - for example, close-in giant planets appear to have fewer (or no) companion planets compared to more distant or smaller planets (Steffen et al. 2012). Insight can be gained into the composition of gas-poor planets by joint modeling in the space of radius, incident flux, and (where available) mass (Figure 2).

Observational follow-up of Kepler targets from the ground and space is underway. Highquality spectra of Kepler host stars will help refine estimates of their properties, and therefore reduce the uncertainty in stellar properties that often dominates the uncertainty in planetary 
properties. A spectrum can also determine the star's projected rotational velocity which, combined with the stellar spin period from Kepler photometry, constrains the angle between the stellar equator and planetary orbit (Hirano et al. 2014). Spectra and adaptive-optics imaging will allow the catalogs to be culled more completely of false positives. The determination of accurate host-star metallicities, which already has provided new insights into the planet-formation process (Buchhave et al. 2012; Wang \& Fischer 2013), will be greatly expanded by LAMOST (Dong et al. 2014). Programs are being developed to follow up some Kepler candidates with large TTVs and establish a longer baseline for TDVs using ground-based telescopes. Kepler stars are among the billions astrometrically monitored by the Gaia mission, launched by ESA in December 2013. Gaia will determine the distances of the Kepler target stars, thereby improving our knowledge of stellar parameters and, consequently, the planetary radii; better radii will in turn improve estimates of the planetary occurrence rates and compositions, and correlations between planetary and stellar properties. For stars within $\sim 200 \mathrm{pc}$, both within and outside the Kepler field, Gaia can detect Jupiter analogues by the astrometric oscillations of their host stars, revealing a more complete architecture for systems in which only the close-in planets are detectable by transits or radial-velocity measurements.

Space-based all-sky surveys, including NASA's Transiting Exoplanet Survey Satellite (TESS; scheduled to launch in 2017) and ESA's PLAnetary Transits and Oscillations of stars (PLATO, scheduled to launch by 2024) could greatly increase the science returns from Kepler by revisiting the Kepler field. Such follow-up would provide a long time baseline that would allow for improved occurrence rates, including an accurate value for $\eta_{\oplus}$, masses for longer period planets from TTVs that would help address outstanding issues about their formation and composition, and the possibility of a substantial number of TDV detections.

Two spacecraft scheduled to be launched in this decade will study known transiting planets. ESA's CHaracterising ExOPlanets Satellite (CHEOPS) will target known exoplanet hosts to discover additional transiting gas-poor planets. NASA's James Webb Space Telescope (JWST) will characterize the atmospheres of gas-poor planets analogous to those that Kepler discovered in abundance; this may break the degeneracy among several composition possibilities.

Since the first handful of exoplanet discoveries two decades ago, the pace of exoplanet research has been extraordinary, driven primarily by ground-based radial-velocity searches of growing power and sophistication and by NASA's Kepler mission. The armada of projects described above will probe new regions of exoplanet parameter space and provide more detailed and accurate probes of the properties of known exoplanets. The challenges for the next two decades will be to maintain the momentum that has been built up in the last two 
decades of exoplanet research, and to work towards the even longer term goal of producing an image of an extrasolar planet comparable to the iconic images of Earth taken by the Apollo astronauts.

Acknowledgments: This research has made use of the Exoplanet Orbit Database at exoplanets.org (Wright et al. 2011) and the Extrasolar Planets Encyclopedia at exoplanets.eu. We are grateful to the Kepler Science Team for their extensive efforts in producing the highquality dataset that has made possible the results reviewed here. We thank William Borucki, Eugene Chiang, Subo Dong, Eve Lee, Eric Lopez, Leslie Rogers, Jason Rowe, Andrew Youdin and Kevin Zahnle for helpful discussions and comments on the manuscript. R.I.D. and S.T. gratefully acknowledge funding from the Miller Institute for Basic Research in Science at the University of California, Berkeley.

\section{REFERENCES}

Albrecht, S., Winn, J. N., Johnson, J. A., et al. 2012, Obliquities of hot Jupiter host stars: evidence for tidal interactions and primordial misalignments, Astrophys. J., 757, id. $18,25 \mathrm{pp}$.

Angerhausen, D., DeLarme, E., \& Morse, J. A. 2014, A comprehensive study of Kepler phase curves and secondary eclipses. Temperatures and albedos of confirmed Kepler giant planets, ArXiv e-prints, arXiv:1404.4348

Ballard, S., Fabrycky, D., Fressin, F., et al. 2011, The Kepler-19 system: a transiting 2.2 $\mathrm{R}_{\oplus}$ planet and a second planet detected via transit timing variations, Astrophys. J., 743, id. 200, 20 pp.

Barclay, T., Rowe, J. F., Lissauer, J. J., et al. 2013, A sub-Mercury-sized exoplanet, Nature, 494, 452-454

Baruteau, C., Crida, A., Paardekooper, S.-J., et al. 2013, Planet-disc interactions and early evolution of planetary systems, ArXiv e-prints, arXiv:1312.4293

Batalha, N. 2014, Exploring exoplanet populations with NASA's Kepler mission, Proceedings of the National Academy of Science, in press

Batalha, N. M., Borucki, W. J., Bryson, S. T., et al. 2011, Kepler's first rocky planet: Kepler-10b, Astrophys. J., 729, id. 27, 21 pp. 
Batalha, N. M., Rowe, J. F., Bryson, S. T., et al. 2013, Planetary candidates observed by Kepler. III. Analysis of the first 16 months of data, Astrophys. J. Suppl., 204, id. 24, $21 \mathrm{pp}$.

Batygin, K. 2012, A primordial origin for misalignments between stellar spin axes and planetary orbits, Nature, 491, 418-420

Borucki, W. J., Koch, D. G., Lissauer, J., et al. 2007, KEPLER mission status, in Astronomical Society of the Pacific Conference Series, Vol. 366, Transiting Extrasolar Planets Workshop, ed. C. Afonso, D. Weldrake, \& T. Henning, 309-319

Borucki, W. J., Koch, D., Basri, G., et al. 2010, Kepler planet-detection mission: introduction and first results, Science, 327, 977-980

Borucki, W. J., Koch, D. G., Basri, G., et al. 2011, Characteristics of planetary candidates observed by Kepler. II. Analysis of the first four months of data, Astrophys. J., 736, id. 19, 22 pp.

Borucki, W. J., Koch, D. G., Batalha, N., et al. 2012, Kepler-22b: a 2.4 Earth-radius planet in the habitable zone of a Sun-like star, Astrophys. J., 745, 120, 16 pp.

Borucki, W. J., Agol, E., Fressin, F., et al. 2013, Kepler-62: a five-planet system with planets of 1.4 and 1.6 Earth radii in the habitable zone, Science, 340, 587-590

Buchhave, L. A., Latham, D. W., Johansen, A., et al. 2012, An abundance of small exoplanets around stars with a wide range of metallicities, Nature, 486, 375-377

Burke, C. J., Bryson, S. T., Mullally, F., et al. 2014, Planetary candidates observed by Kepler. IV. Planet sample from Q1-Q8 (22 months), Astrophys. J. Suppl., 210, id. 19, $12 \mathrm{pp}$.

Carter, J. A., Agol, E., Chaplin, W. J., et al. 2012, Kepler-36: a pair of planets with neighboring orbits and dissimilar densities, Science, 337, 556-559

Chaplin, W. J., \& Miglio, A. 2013, Asteroseismology of solar-type and red-giant stars, ARA\&A, 51, 353-392

Chiang, E., \& Laughlin, G. 2013, The minimum-mass extrasolar nebula: in situ formation of close-in super-Earths, Mon. Not. R. Astron. Soc., 431, 3444-3455

Christensen-Dalsgaard, J. 2013, The new era of asteroseismology, in EAS Publications Series, Vol. 63, 91-104 
Christiansen, J. L., Clarke, B. D., Burke, C. J., et al. 2013, Measuring transit signal recovery in the Kepler pipeline. I. Individual events, Astrophys. J. Suppl., 207, id. 35, 12 pp.

Dawson, R. I., \& Johnson, J. A. 2012, The photoeccentric effect and proto-hot Jupiters. I. Measuring photometric eccentricities of individual transiting planets, Astrophys. J., 756, id. 122,13 pp.

Dawson, R. I., \& Murray-Clay, R. A. 2013, Giant planets orbiting metal-rich stars show signatures of planet-planet interactions, Astrophys. J., 767, id. L24, 6 pp.

Dawson, R. I., Johnson, J. A., Fabrycky, D. C., et al. 2014, Large Eccentricity, Low Mutual Inclination: The Three-dimensional Architecture of a Hierarchical System of Giant Planets, ApJ, 791, 89

Deck, K. M., Holman, M. J., Agol, E., et al. 2012, Rapid dynamical chaos in an exoplanetary system, Astrophys. J., 755, id. L21, 7 pp. Erratum at ApJ 774, id. L15

Demory, B.-O. 2014, The albedos of Kepler's close-in super-Earths, Astrophys. J., 789, id. L20, 6 pp.

Dong, S., \& Zhu, Z. 2013, Fast rise of "Neptune-size" planets (4-8 $\mathrm{R}_{\oplus}$ ) from $P \sim 10$ to $\sim 250$ days. Statistics of Kepler planet candidates up to $\sim 0.75$ AU, Astrophys. J., 778, id. $53,11 \mathrm{pp}$.

Dong, S., Zheng, Z., Zhu, Z., et al. 2014, On the metallicities of Kepler stars, Astrophys. J., 789, id. L3, 4 pp.

Doyle, L. R., Carter, J. A., Fabrycky, D. C., et al. 2011, Kepler-16: a transiting circumbinary planet, Science, 333, 1602-1606

Dreizler, S., \& Ofir, A. 2014, Kepler-9 revisited: 60\% the mass with six times more data, ArXiv e-prints, arXiv:1403.1372

Dressing, C. D., \& Charbonneau, D. 2013, The occurrence rate of small planets around small stars, Astrophys. J., 767, id. 95, 20 pp.

Eggenberger, A. 2010, Detection and characterization of planets in binary and multiple systems, in EAS Publications Series, ed. K. Goździewski, A. Niedzielski, \& J. Schneider, Vol. 42, 19-37

Evans, N. W., \& Tabachnik, S. A. 2002, Structure of possible long-lived asteroid belts, Mon. Not. R. Astron. Soc., 333, L1-L5 
Fabrycky, D., \& Tremaine, S. 2007, Shrinking binary and planetary orbits by Kozai cycles with tidal friction, Astrophys. J., 669, 1298-1315

Fabrycky, D. C., Lissauer, J. J., Ragozzine, D., et al. 2014, Architecture of Kepler's Multitransiting Systems. II. New Investigations with Twice as Many Candidates, ApJ, 790, id. 146,12 pp.

Fang, J., \& Margot, J.-L. 2013, Are planetary systems filled to capacity? A study based on Kepler results, Astrophys. J., 767, id. 115, 7 pp.

Fischer, D. A., \& Valenti, J. 2005, The planet-metallicity correlation, Astrophys. J., 622, 1102-1117

Fischer, D. A., Schwamb, M. E., Schawinski, K., et al. 2012, Planet Hunters: the first two planet candidates identified by the public using the Kepler public archive data, Mon. Not. R. Astron. Soc., 419, 2900-2911

Foreman-Mackey, D., Hogg, D. W., \& Morton, T. D. 2014, Exoplanet population inference and the abundance of Earth analogs from noisy, incomplete catalogs, ArXiv e-prints, arXiv:1406.3020

Fortney, J. J., Marley, M. S., \& Barnes, J. W. 2007, Planetary radii across five orders of magnitude in mass and stellar insolation: application to transits, Astrophys. J., 659, 1661-1672

Fressin, F., Torres, G., Rowe, J. F., et al. 2012, Two Earth-sized planets orbiting Kepler-20, Nature, 482, 195-198

Fressin, F., Torres, G., Charbonneau, D., et al. 2013, The false positive rate of Kepler and the occurrence of planets, Astrophys. J., 766, id. 81, 20 pp.

Gilliland, R. L., Chaplin, W. J., Dunham, E. W., et al. 2011, Kepler mission stellar and instrument noise properties, Astrophys. J. Suppl., 197, id. 6, 19 pp.

Gladman, B. 1993, Dynamics of systems of two close planets, Icarus, 106, 247-263

Goldreich, P., \& Schlichting, H. E. 2014, Overstable librations can account for the paucity of mean motion resonances among exoplanet pairs, Astron. J., 147, id. 32, 11 pp.

Goldreich, P., \& Tremaine, S. 1980, Disk-satellite interactions, Astrophys. J., 241, 425-441

Hadden, S., \& Lithwick, Y. 2014, Densities and eccentricities of 139 Kepler planets from transit time variations, Astrophys. J., 787, id. 80, 7 pp. 
Hansen, B. M. S., \& Murray, N. 2013, Testing in situ assembly with the Kepler planet candidate sample, Astrophys. J., 775, id. 53, 17 pp.

Heller, C. H. 1993, Encounters with protostellar disks. I. Disk tilt and the nonzero solar obliquity, Astrophys. J., 408, 337-346

Hirano, T., Sanchis-Ojeda, R., Takeda, Y., et al. 2014, Measurements of stellar inclinations for Kepler planet candidates. II. Candidate spin-orbit misalignments in single- and multiple-transiting systems, Astrophys. J., 783, id. 9, 10 pp.

Holman, M. J. 1997, A possible long-lived belt of objects between Uranus and Neptune, Nature, 387, 785-788

Holman, M. J., \& Wiegert, P. A. 1999, Long-term stability of planets in binary systems, Astron. J., 117, 621-628

Holman, M. J., et al. 2010, Kepler-9: a system of multiple planets transiting a Sun-like star, confirmed by timing variations, Science, 330, 51-54

Howard, A. W., Marcy, G. W., Bryson, S. T., et al. 2012, Planet occurrence within 0.25 AU of solar-type stars from Kepler, Astrophys. J. Suppl., 201, id. 15, 20 pp.

Howard, A. W., Sanchis-Ojeda, R., Marcy, G. W., et al. 2013, A rocky composition for an Earth-sized exoplanet, Nature, 503, 381-384

Ida, S. 1990, Stirring and dynamical friction rates of planetesimals in the solar gravitational field, Icarus, 88, 129-145

Jenkins, J. M., Caldwell, D. A., Chandrasekaran, H., et al. 2010, Overview of the Kepler Science Processing Pipeline, Astrophys. J., 713, L87-L91

Jontof-Hutter, D., Lissauer, J. J., Rowe, J. F., \& Fabrycky, D. C. 2014, Kepler-79's low density planets, Astrophys. J., 785, id. 15, 14 pp.

Kipping, D. M. 2014, Characterizing distant worlds with asterodensity profiling, Mon. Not. R. Astron. Soc., 440, 2164-2184

Kipping, D. M., Bakos, G. Á., Buchhave, L., Nesvorný, D., \& Schmitt, A. 2012a, The Hunt for Exomoons with Kepler (HEK). I. Description of a new observational project, Astrophys. J., 750, id. 115, 19 pp.

Kipping, D. M., Dunn, W. R., Jasinski, J. M., \& Manthri, V. P. 2012b, A novel method to photometrically constrain orbital eccentricities: multibody asterodensity profiling, Mon. Not. R. Astron. Soc., 421, 1166-1188 
Kipping, D. M., Hartman, J., Buchhave, L. A., et al. 2013, The hunt for exomoons with Kepler (HEK). II. Analysis of seven viable satellite-hosting planet candidates, Astrophys. J., 770, id. 101, 30 pp.

Koch, D. G., Borucki, W. J., Basri, G., et al. 2010, Kepler mission design, realized photometric performance, and early science, Astrophys. J., 713, L79-L86

Kopparapu, R. K., Ramirez, R., Kasting, J. F., et al. 2013, Habitable zones around mainsequence stars: new estimates, Astrophys. J., 765, id. 131, 16 pp. Erratum at ApJ 770 , id. 82

Latham, D. W., Rowe, J. F., Quinn, S. N., et al. 2011, A first comparison of Kepler planet candidates in single and multiple systems, Astrophys. J., 732, id. L24, 4 pp.

Lissauer, J. J., Fabrycky, D. C., Ford, E. B., et al. 2011a, A closely packed system of lowmass, low-density planets transiting Kepler-11, Nature, 470, 53-58

Lissauer, J. J., Ragozzine, D., Fabrycky, D. C., et al. 2011b, Architecture and dynamics of Kepler's candidate multiple transiting planet systems, Astrophys. J. Suppl., 197, id. 8, $26 \mathrm{pp}$.

Lissauer, J. J., Marcy, G. W., Rowe, J. F., et al. 2012, Almost all of Kepler's multiple-planet candidates are planets, Astrophys. J., 750, id. 112, 15 pp.

Lissauer, J. J., Jontof-Hutter, D., Rowe, J. F., et al. 2013, All six planets known to orbit Kepler-11 have low densities, Astrophys. J., 770, id. 131, 15 pp.

Lissauer, J. J., Marcy, G. W., Bryson, S. T., et al. 2014, Validation of Kepler's multiple planet candidates. II. Refined statistical framework and descriptions of systems of special interest, Astrophys. J., 784, id. 44, 21 pp.

Lithwick, Y., \& Wu, Y. 2012, Resonant repulsion of Kepler planet pairs, Astrophys. J., 756, id. L11, 5 pp.

Lithwick, Y., Xie, J., \& Wu, Y. 2012, Extracting planet mass and eccentricity from TTV data, Astrophys. J., 761, id. 122, 11 pp.

Lopez, E. D., \& Fortney, J. J. 2013, The role of core mass in controlling evaporation: the Kepler radius distribution and the Kepler-36 density dichotomy, Astrophys. J., 776, id. 2, 11 pp.

- 2014, Understanding the mass-radius relation for sub-Neptunes: radius as a proxy for composition, ApJ, 792, 1 
Lopez, E. D., Fortney, J. J., \& Miller, N. 2012, How thermal evolution and mass-loss sculpt populations of super-earths and sub-neptunes: application to the Kepler-11 system and beyond, Astrophys. J., 761, id. 59, 13 pp.

Marcy, G. W., Isaacson, H., Howard, A. W., et al. 2014, Masses, radii, and orbits of small Kepler planets: the transition from gaseous to rocky planets, Astrophys. J. Suppl., 210, id. 20, 70 pp.

Moorhead, A. V., Ford, E. B., Morehead, R. C., et al. 2011, The distribution of transit durations for Kepler planet candidates and implications for their orbital eccentricities, Astrophys. J. Suppl., 197, id. 1, 15 pp.

Morton, T. D. 2012, An efficient automated validation procedure for exoplanet transit candidates, Astrophys. J., 761, id. 6, 12 pp.

Morton, T. D., \& Johnson, J. A. 2011, On the low false positive probabilities of Kepler planet candidates, Astrophys. J., 738, id. 170, 12 pp.

Morton, T. D., \& Swift, J. 2014, The Radius Distribution of Planets around Cool Stars, ApJ, 791, 10

Muirhead, P. S., Johnson, J. A., Apps, K., et al. 2012, Characterizing the cool KOIs. III. KOI 961: a small star with large proper motion and three small planets, Astrophys. J., 747 , id. 144, 16 pp.

Nesvorný, D., Kipping, D. M., Buchhave, L. A., et al. 2012, The detection and characterization of a nontransiting planet by transit timing variations, Science, 336, 1133-1136

Orosz, J. A., Welsh, W. F., Carter, J. A., et al. 2012, Kepler-47: a transiting circumbinary multiplanet system, Science, 337, 1511-1514

Peale, S. J. 1976, Orbital resonances in the solar system, ARA\&A, 14, 215-246

Pepe, F., Cameron, A. C., Latham, D. W., et al. 2013, An Earth-sized planet with an Earth-like density, Nature, 503, 377-380

Petigura, E. A., Howard, A. W., \& Marcy, G. W. 2013a, Prevalence of Earth-size planets orbiting Sun-like stars, Proceedings of the National Academy of Science, 110, 1927319278

Petigura, E. A., \& Marcy, G. W. 2012, Identification and removal of noise modes in Kepler photometry, Publications of the Astronomical Society of the Pacific, 124, 1073-1082 
Petigura, E. A., Marcy, G. W., \& Howard, A. W. 2013b, A plateau in the planet population below twice the size of Earth, Astrophys. J., 770, id. 69, 21 pp.

Petrovich, C., Malhotra, R., \& Tremaine, S. 2013, Planets near mean-motion resonances, Astrophys. J., 770, id. 24, 16 pp.

Petrovich, C., Tremaine, S., \& Rafikov, R. 2014, Scattering outcomes of close-in planets: constraints on planet migration, Astrophys. J., 786, id. 101, 10 pp.

Plavchan, P., Bilinski, C., \& Currie, T. 2014, Investigation of Kepler Objects of Interest stellar parameters from observed transit durations, Publications of the Astronomical Society of the Pacific, 126, 34-47

Prsa, A., Batalha, N., Slawson, R. W., et al. 2011, Kepler eclipsing binary stars. I. Cata$\log$ and principal characterization of 1879 eclipsing binaries in the first data release, Astron. J., 141, id. 83, 16 pp.

Rafikov, R. R., \& Slepian, Z. S. 2010, Dynamical evolution of thin dispersion-dominated planetesimal disks, Astron. J., 139, 565-579

Raghavan, D., McAlister, H. A., Henry, T. J., et al. 2010, A survey of stellar families: multiplicity of solar-type stars, Astrophys. J. Suppl., 190, 1-42

Rappaport, S., Levine, A., Chiang, E., et al. 2012, Possible disintegrating short-period superMercury orbiting KIC 12557548, Astrophys. J., 752, id. 1, 13 pp.

Rein, H. 2012, Period ratios in multiplanetary systems discovered by Kepler are consistent with planet migration, Mon. Not. R. Astron. Soc., 427, L21-L24

Ricker, G. R., Winn, J. N., Vanderspek, R., et al. 2014, The Transiting Exoplanet Survey Satellite mission, Journal of the American Association of Variable Star Observers (JAAVSO), 42, 234

Rogers, L. 2014, Most 1.6 Earth-radii planets are not rocky, ArXiv e-prints, arXiv:1407.4457

Rogers, L. A., Bodenheimer, P., Lissauer, J. J., \& Seager, S. 2011, Formation and structure of low-density exo-Neptunes, Astrophys. J., 738, id. 59, 16 pp.

Sanchis-Ojeda, R., Rappaport, S., Winn, J. N., et al. 2014, A study of the shortest-period planets found with Kepler, Astrophys. J., 787, id. 47, 18 pp.

-. 2013, Transits and occultations of an Earth-sized planet in an $8.5 \mathrm{hr}$ orbit, Astrophys. J., 774, id. 54, 9 pp. Erratum at ApJ 783, id. 64 
Sanchis-Ojeda, R., Fabrycky, D. C., Winn, J. N., et al. 2012, Alignment of the stellar spin with the orbits of a three-planet system, Nature, 487, 449-453

Santerne, A., Díaz, R. F., Moutou, C., et al. 2012, SOPHIE velocimetry of Kepler transit candidates. VII. A false-positive rate of $35 \%$ for Kepler close-in giant candidates, Astron. Astrophys., 545, id. A76, 16 pp.

Santos, N. C., Israelian, G., \& Mayor, M. 2001, The metal-rich nature of stars with planets, Astron. Astrophys., 373, 1019-1031

- 2004, Spectroscopic [Fe/H] for 98 extra-solar planet-host stars. Exploring the probability of planet formation, Astron. Astrophys., 415, 1153-1166

Schlaufman, K. C. 2014, Tests of in situ Formation Scenarios for Compact Multiplanet Systems, ApJ, 790, 91

Smith, A. W., \& Lissauer, J. J. 2009, Orbital stability of systems of closely-spaced planets, Icarus, 201, 381-394

Smith, J. C., Stumpe, M. C., Van Cleve, J. E., et al. 2012, Kepler presearch data conditioning. II. A Bayesian approach to systematic error correction, Publications of the Astronomical Society of the Pacific, 124, 1000-1014

Sousa, S. G., Santos, N. C., Israelian, G., Mayor, M., \& Udry, S. 2011, Spectroscopic stellar parameters for 582 FGK stars in the HARPS volume-limited sample. Revising the metallicity-planet correlation, Astron. Astrophys., 533, id. A141, 9 pp.

Steffen, J. H., Ragozzine, D., Fabrycky, D. C., et al. 2012, Kepler constraints on planets near hot Jupiters, Proceedings of the National Academy of Science, 109, 7982-7987

Stumpe, M. C., Smith, J. C., Van Cleve, J. E., et al. 2012, Kepler presearch data conditioning. I. Architecture and algorithms for error correction in Kepler Light Curves, Publications of the Astronomical Society of the Pacific, 124, 985-999

Swift, J. J., Johnson, J. A., Morton, T. D., et al. 2013, Characterizing the cool KOIs. IV. Kepler-32 as a prototype for the formation of compact planetary systems throughout the Galaxy, Astrophys. J., 764, id. 105, 14 pp.

Tabachnik, S., \& Tremaine, S. 2002, Maximum-likelihood method for estimating the mass and period distributions of extrasolar planets, Mon. Not. R. Astron. Soc., 335, 151158 
Tamuz, O., Ségransan, D., Udry, S., et al. 2008, The CORALIE survey for southern extrasolar planets. XV. Discovery of two eccentric planets orbiting HD 4113 and HD 156846 , Astron. Astrophys., 480, L33-L36

Tenenbaum, P., Christiansen, J. L., Jenkins, J. M., et al. 2012, Detection of potential transit signals in the first three quarters of Kepler mission data, Astrophys. J. Suppl., 199, id. $24,15 \mathrm{pp}$.

Torres, G., Fressin, F., Batalha, N. M., et al. 2011, Modeling Kepler transit light curves as false positives: rejection of blend scenarios for Kepler-9, and validation of Kepler-9 d, a super-Earth-size planet in a multiple system, ApJ, 727, id. 24, 18 pp.

Tremaine, S. 1991, On the origin of the obliquities of the outer planets, Icarus, 89, 85-92

Tremaine, S., \& Dong, S. 2012, The statistics of multi-planet systems, Astron. J., 143, id. 94, $16 \mathrm{pp}$.

Wang, J., \& Fischer, D. A. 2013, The metal-rich stars get richer in planets for all but planets with $R_{p} \leq 2 R_{E}$, ArXiv e-prints, arXiv:1310.7830

Wang, J., Fischer, D. A., Xie, J.-W., \& Ciardi, D. R. 2014, Influence of Stellar Multiplicity on Planet Formation. II. Planets are Less Common in Multiple-star Systems with Separations Smaller than 1500 AU, ApJ, 791, 111

Weiss, L. M., \& Marcy, G. W. 2014, The mass-radius relation for 65 exoplanets smaller than 4 Earth radii, Astrophys. J., 783, id. L6, 7 pp.

Winn, J. N., Albrecht, S., Johnson, J. A., et al. 2011, Spin-orbit alignment for the circumbinary planet host Kepler-16 A, Astrophys. J., 741, id. L1, 6 pp.

Wright, J. T., Marcy, G. W., Howard, A. W., et al. 2012, The frequency of hot Jupiters orbiting nearby solar-type stars, Astrophys. J., 753, id. 160, 5 pp.

Wright, J. T., Fakhouri, O., Marcy, G. W., et al. 2011, The Exoplanet Orbit Database, Publications of the Astronomical Society of the Pacific, 123, 412-422

Wu, Y., \& Lithwick, Y. 2013, Density and eccentricity of Kepler planets, Astrophys. J., 772, id. 74,13 pp.

Youdin, A. N. 2011, The exoplanet census: a general method applied to Kepler, Astrophys. J., 742, id. 38, 13 pp. 
Zakamska, N. L., Pan, M., \& Ford, E. B. 2011, Observational biases in determining extrasolar planet eccentricities in single-planet systems, Mon. Not. R. Astron. Soc., 410, 18951910 